\title{
Using neural networks to improve simulations in the gray zone
}

\author{
Raphael Kriegmair ${ }^{1}$, Yvonne Ruckstuhl ${ }^{1}$, Stephan Rasp ${ }^{2}$, and George Craig ${ }^{1}$ \\ ${ }^{1}$ Meteorological Institute Munich, Ludwig-Maximilians-Universität München, Germany \\ ${ }^{2}$ ClimateAi, Inc.
}

Correspondence: Yvonne Ruckstuhl (yvonne.ruckstuhl@lmu.de)

\begin{abstract}
Machine learning represents a potential method to cope with the gray zone problem of representing motions in dynamical systems on scales comparable to the model resolution. Here we explore the possibility of using a neural network to directly learn the error caused by unresolved scales. We use a modified shallow water model which includes highly nonlinear processes mimicking atmospheric convection. To create the training dataset we run the model in a high and a low-resolution

5 setup and compare the difference after one low resolution time step starting from the same initial conditions, thereby obtaining an exact target. The neural network is able to learn a large portion of the difference when evaluated "offline" on a validation set. When coupled to the low-resolution model, we find large forecast improvements up to one day on average. After this, the accumulated error due to the mass conservation violation of the neural network starts to dominate and deteriorates the forecast. This deterioration can effectively be delayed by adding a penalty term to the loss function used to train the ANN to conserve mass in a weak sense. This study reinforces the need to include physical constraints in neural network parameterizations.
\end{abstract}

Copyright statement. TEXT

\section{Introduction}

Current limitations on computational power force weather and climate prediction to use relatively low resolution simulations. Subgrid scale processes, i.e. processes that are not resolved by the model grid, are typically represented using physical parameterizations (Stensrud, 2009). Inaccuracies in these parameterizations are known to cause errors in weather forecasts and biases in climate projections. While parameterizations are becoming more sophisticated over time, there is evidence that key structural uncertainties remain (Randall et al., 2003; Randall, 2013; Jones and Randall, 2011).

A particularly difficult problem in the representation of unresolved processes is the so-called gray zone (Chow et al., 2019; Honnert et al., 2020), where a certain physical phenomenon such as a cumulus cloud is similar in size to the model resolution and hence partially resolved. In the development of many classical parameterizations, features are assumed to be small in comparison to the model resolution. This scale separation provides a conceptual basis for specifying the average effects of the unresolved flow features on the resolved flow. In contrast, there is no theoretical basis for determining such a relationship in the gray zone. Instead, the truncation error of the numerical model is a significant factor. While we might still expect there to be some relationship between the resolved and unresolved parts of the flow, we have no way to define it. 

prediction are in the gray zone for cumulus convection. These models are typically run without a parameterization for deep convection, but the resolution of 2-4 km does not give accurate results for typical convective cloud structures are often less than $10 \mathrm{~km}$ in size (Bryan et al., 2003; Wagner et al., 2018). The flow on these scales is also influenced by partially-resolved orography and other surface properties, which also belong to the gray zone. With no obvious methodology for developing a parameterization suitable for these scales, the most that can be hoped for current schemes is that their influence diminishes with increasing resolution (Jeworrek et al., 2019).

Using machine learning methods such as artificial neural networks (ANNs) for alleviating the problems described above has received increasing attention over the past years. One approach is to avoid the need of paramaterizations all together by emulating the entire model using observations (Brunton et al., 2016; Pathak et al., 2018; Faranda et al., 2020; Fablet et al., 2018; Scher, 2018; Dueben and Bauer, 2018). In these studies a dense and noise free observation network is often assumed. Brajard et al. (2020a) and Bocquet et al. (2020) circumvent the requirement of this assumption by using data assimilation to form targets for ANNs from sparse and noisy observations.

Though studies have shown that surrogate models produced by machine learning can be accurate for small dynamical systems, replacing an entire numerical weather prediction model for operational use is not yet within our reach. Therefore, a more practical approach is to use ANNs as replacement for uncertain parameterizations. This has been done either by learning from physics based expensive parametrization schemes (O’Gorman and Dwyer, 2018; Rasp et al., 2018) or high resolution simulations (Krasnopolsky et al., 2013; Brenowitz and Bretherton, 2019; Bolton and Zanna, 2019; Rasp, 2020; Yuval and O'Gorman, 2020), which is the approach we take here. Such data driven techniques could be a way to reduce the structural uncertainty of traditional parameterizations, even at gray zone resolutions where the physical basis of the parameterization is no longer valid. The first challenge is to create the training data, i.e. to separate the resolved and unresolved scales from the high-resolution simulation. Brenowitz and Bretherton (2019) use a coarse-graining approach based on subtracting the coarse grained advection term from the local tendencies. This approach can be used for any model and resolution but is sensitive to the choice of grid and time step. Further, the resulting subgrid tendencies are only an approximation and may not represent the real difference between the low and high-resolution model. Yuval and O'Gorman (2020) use the same model version for low and high-resolution simulations and compute exact differences after a single low-resolution time step by starting both model versions from the same initial conditions. They manage to obtain stable long-term simulations using the low-resolution model with a machine learning correction that come close the the high-resolution ground truth.

Here, we use the modified rotating shallow water (modRSW) model to explore the use of a machine learning subgrid representation in a highly non-linear dynamical system. The modRSW is an idealized fluid model of convective-scale numerical weather prediction, in which convection is triggered by orography. As such, the model mimics the gray zone problem of operational kilometer-scale models. Using a simplified model allows us to focus on some key conceptual questions surrounding machine learning parameterizations, such as how choices in neural network training affect long-term physical consistency. In particular, we include weak physical constraints in the training procedure. 
The contents of this work are outlined in the following. Section 2 introduces the experiment setup used to obtain and analyze results. The modRSW model is briefly explained in Section 2.1, followed by a description of the training data generation in Section 2.2. The architecture and training process of the ANN used in this research are given in 2.3. Results are presented in Section 3. followed by a conclusion in Section 4.

\section{Experiment setup}

\subsection{The modRSW Model}

65 The modRSW model (Kent et al., 2017) used in this research represents an extended version of the 1D shallow water equations, i.e. 1D fluid flow over orography. Its prognostic variables are fluid height $h$, wind speed $u$ and a rain mass fraction $r$. Based on the model by Würsch and Craig (Würsch and Craig, 2014) it implements two threshold heights, $H_{c}<H_{r}$, initiating convection and rain production, respectively. Convection is stimulated by modifying the pressure term to remain constant where $h$ rises above $H_{c}$. In contrast to Würsch and Craig (2014), the modRSW model does not apply diffusion or stochastic forcing. The model is mass conserving, meaning that the domain mean of $h$ is constant over time. In this study, a small but significant modelintrinsic drift in the domain mean of $u$ is accounted for by adding a relaxation term. This term is defined using a corresponding time scale $t_{\text {relax }}$, as $\left(\bar{u}_{0}-\bar{u}_{t}\right) \cdot t_{\text {relax }}$. Depending on the orography used, this model yields a continuous range of dynamical organization between regular and chaotic behaviour. We pick one simulation from each extreme and compare results to identify general and flow dependent aspects.

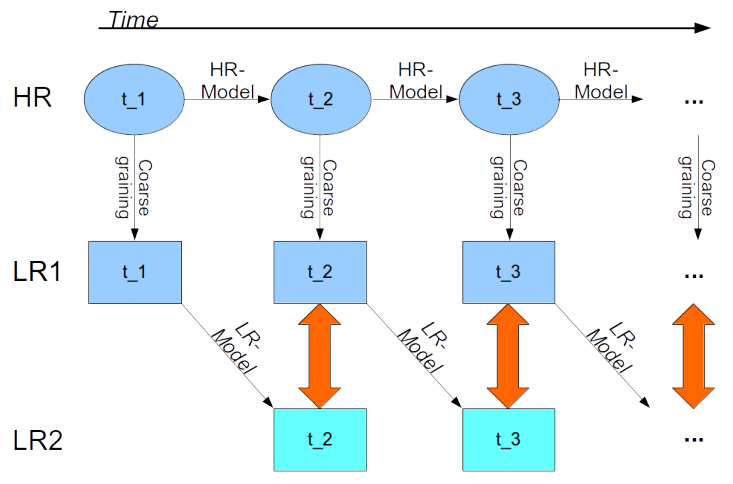

Figure 1. Schematic of training data generation process. A HR run is coarse grained to LR to generate model truth. Each model truth state is integrated forward for one time step using LR dynamics. The difference between the obtained states and corresponding model truth defines the desired network output (red arrows), while the preceding model truth defines the network input. 


\subsection{Training Data Generation}

Conceptually, the ANN's task is to correct a low resolution (LR) model forecast towards the model truth, which is a coarse grained high resolution (HR) model simulation. The coarse graining factor in this study is set to 4. A training sample (inputtarget pair) is defined by the model truth at some time $t_{n}$ and the difference between the model truth and the corresponding LR forecast at $t_{n+1}=t_{n}+d t$, respectively (see Figure 1). To generate the model truth, HR data are obtained by integrating the modRSW model forward using the parameters shown in Table 1.

\begin{tabular}{|c|c|c|c|}
\hline Model Parameter & Symbol & Value & Notes \\
\hline HR gridpoint number & $H R$ & 800 & - \\
\hline LR gridpoint number & $L R$ & 200 & - \\
\hline Time step & $d t$ & 0.001 & - \\
\hline Domain size (non dim.) & $L$ & 1.0 & - \\
\hline CFL & - & 0.5 & - \\
\hline Convection threshold & $H_{c}$ & 1.02 & - \\
\hline Rain threshold & $H_{r}$ & 1.05 & - \\
\hline Initial total height & $H_{0}$ & 1.0 & - \\
\hline Rossby Number & Ro & $\infty$ & - \\
\hline Froude Number & $F r$ & 1.1 & - \\
\hline Effective gravity & $g$ & $F r^{-2}$ & - \\
\hline Beta & $\beta$ & 0.2 & - \\
\hline Alpha & $\alpha^{2}$ & 10 & - \\
\hline Rain Conversion Factor & $c^{2}$ & $0.1 \times g \times H_{r}$ & - \\
\hline Wind Relaxation time scale & $t_{\text {relax }}$ & $d t$ & - \\
\hline \multicolumn{4}{|l|}{ Orography Generation } \\
\hline Maximum wave number & $k_{\max }$ & 100 & - \\
\hline Maximum Amplitude & $B_{\max }$ & 0.1 & - \\
\hline
\end{tabular}

Table 1. Model setting parameters are given as $A(k)=1 / k$, while phase shifts for each term are randomly chosen from $[0, L]$. All states and the orography are subsequently coarse grained to LR, resulting in model truth (LR1). Each LR1 state is integrated forward for a single timestep using the modRSW model on LR with the coarse grained orography, resulting in a single step prediction (LR2). The synchronized differences $L R 1\left(t_{i}\right)-L R 2\left(t_{i}\right)$ then define the training targets corresponding to the input $L R 1\left(t_{i-1}\right)$, which 
includes the orography. A time series of $T=200000$ time steps is generated for both orographies, of which the odd time steps are used for training and the even time steps for validation.

\subsection{Convolutional ANN}

A characteristic property of convolutional ANNs is that they reflect spatial invariance and localization. These two properties also apply to the dynamics of many physical systems, such as the one investigated here. They differ from e.g. dense networks by the use of a so called kernel. This vector is "moved" step by step across the domain grid, covering $k$ grid points at each position. At each position, the dot product of kernel and current grid values is computed, determining (along with an activation function) the corresponding output value.

The ANN structure used in this research is described in the following. 5 hidden layers are applied, each using the $\operatorname{ReLU}$ activation function. The input layer uses $R e L U$ as well, while the output layer uses a linear activation function. All hidden layers have 32 filters. The input and output layer shapes are defined by input and target data. The kernel size is set uniformly to 3 grid points. Biases are applied throughout the ANN.

The loss is determined during training by comparing the ANN output to the corresponding target. A standard measure for loss is the mean squared error (MSE). However, any loss function can be used to tailor to the application. For example, additional terms can be added to impose weak constraints on the training process, as for example done in Ruckstuhl et al. (2021). This possibility is exploited here to impose mass conservation in a weak sense. The constraint is implemented by penalizing the deviation of the square of domain mean $h$ corrections from zero. The loss function is defined as

$$
M S E\left(y_{\text {out }}, y_{\text {target }}\right)+w_{\text {mass }} \cdot(\overline{\Delta h})^{2}
$$

where the second term represents a weighted mass conservation constraint. In this expression, $M S E$ denotes the mean square error, $w_{\text {mass }}$ is the mass conservation constraint weighting, $\Delta h$ are ANN corrections for $h$ and the overbar denotes the domain mean.

The Adam algorithm with a learning rate of $10^{-3}$ is used to minimize the loss function over the ANN weights in batches of 256 samples. Since the loss function is typically not convex, the ANN likely converges to a local minimum. To sample this error, we repeat the training of each ANN presented in this paper with different initial weights 5 times. The initial weights are drawn randomly. For all ANNs a total of 1000 epochs is performed.

\section{Results}

We performed a series of experiments designed to investigate the feasibility of using an ANN to correct for model error due to unresolved scales. In section 3.1 we first explore the performance of the ANNs trained with the standard loss function, the MSE. Next, the weak constraint is added to the loss function as in equation (1) and the benefits are examined in section 3.2. 

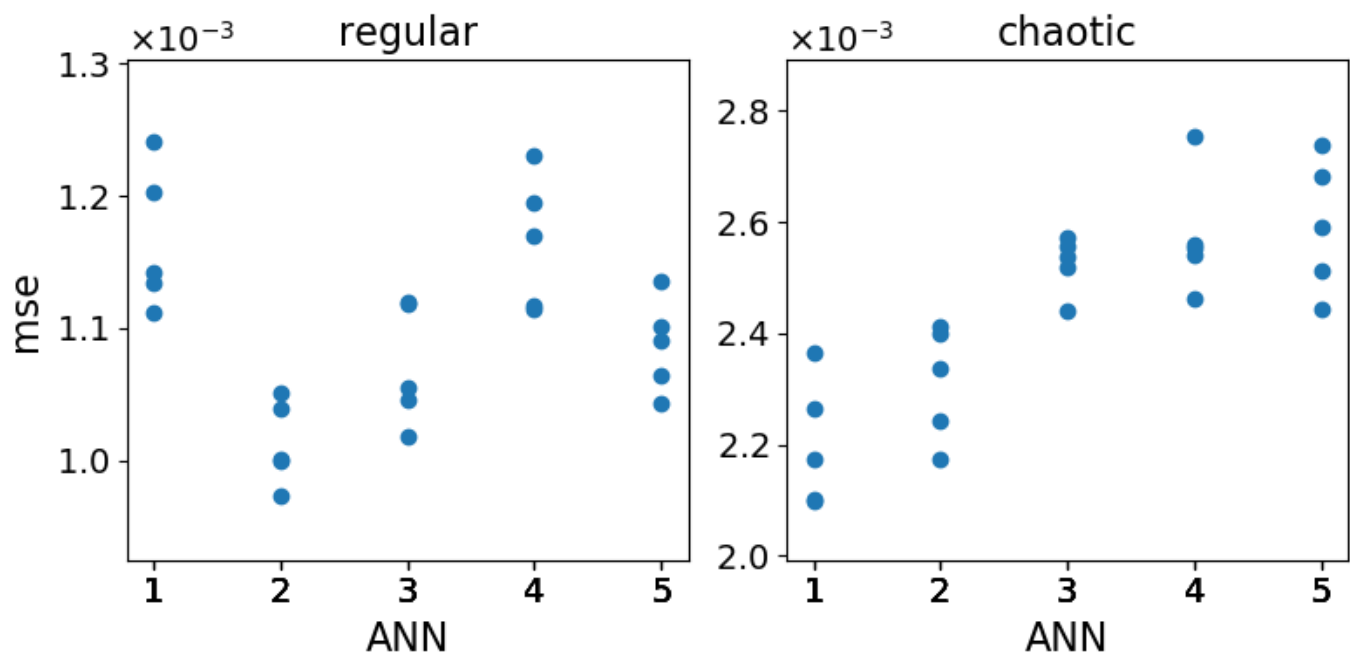

Figure 2. Loss function value (mse) of validation data corresponding to the last 5 epochs of the training process (y-axis) for each trained ANN (x-axis).

\subsection{ANN with standard loss function}

Figure 2 illustrates the variability among the 5 ANNs with different initial training weights, versus the variability among the last 5 epochs of each ANN for both orographies. Since neither the ANN nor the epoch number seems to dominate the variability of the loss value, we use both to sample the total ANN variability, resulting in $5 \times 5=25$ samples for each ANN training setup that is presented in the remainder of this paper.
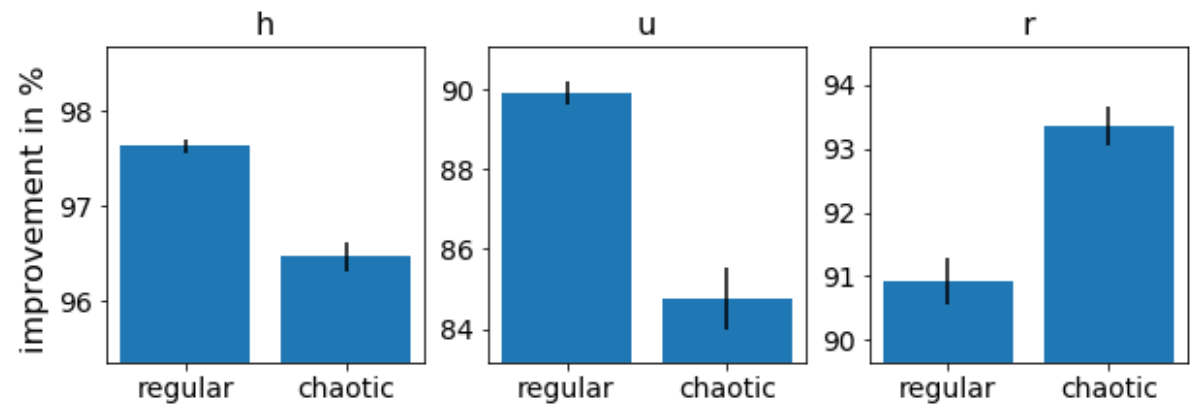

Figure 3. Mean (bars) and standard deviation (error bars) of the RMSE of ANN corrected single time step predictions of the validation data with respect to the model truth, expressed as improvements in percentage with respect to the RMSE of the corresponding uncorrected LR single time step predictions, for the regular and chaotic case (y-axis) and for variables $h$ (left), $u$ (middle) and $r$ (right). 
Figure 3 shows the improvement of single time step predictions when the trained ANNs are applied, calculated from root mean squared errors (RMSE). The bars show the the averaged RMSE of the prediction after the ANN is applied divided by the average RMSE of the prediction before the ANN is applied times 100. The error bars indicate the standard deviation over the 25 samples. The improvements are large for both the chaotic and regular case.
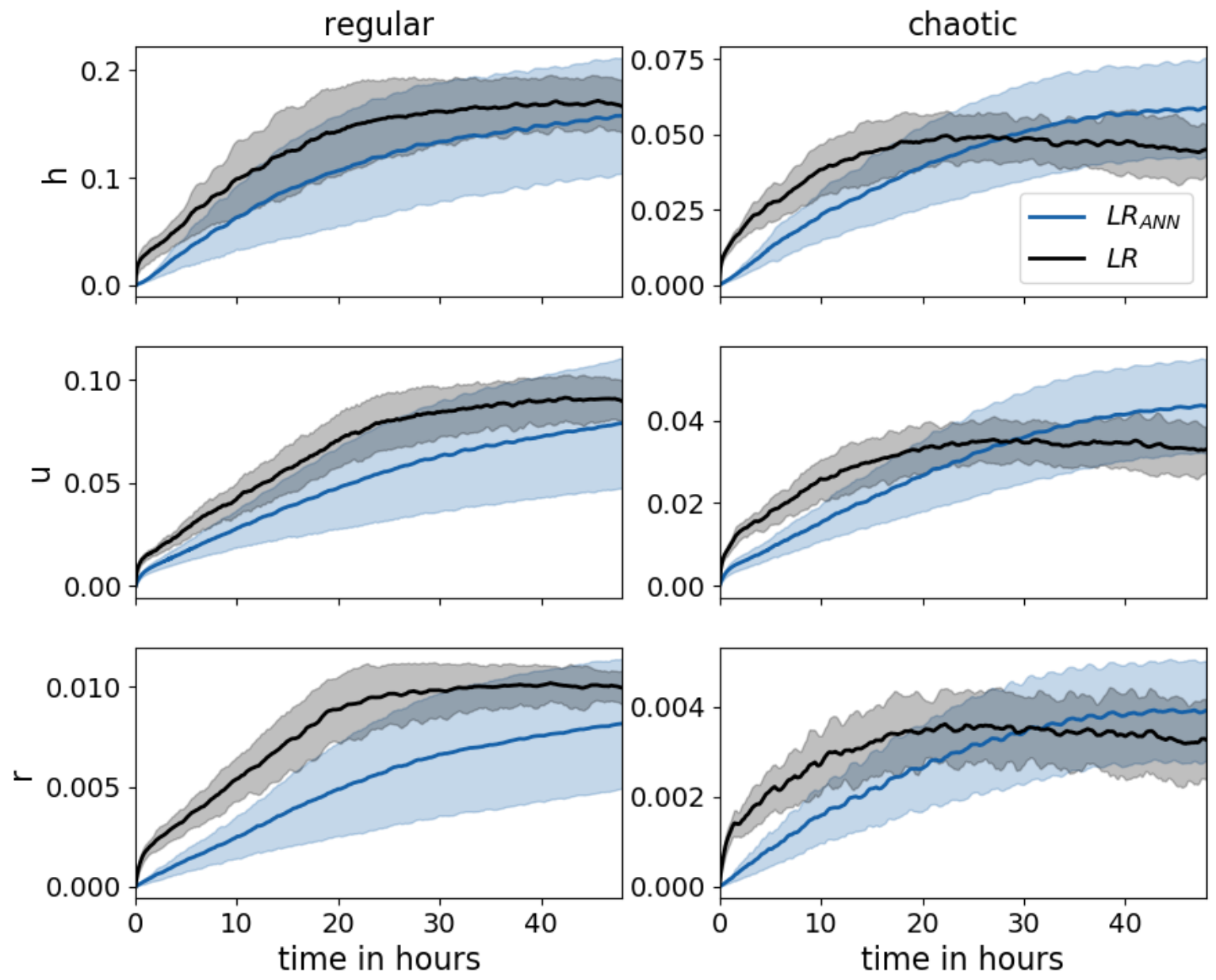

Figure 4. RMSE evolution of 48-hour forecasts for the respective model variables of $L R$ (black) and $L R_{A N N}$ (blue), averaged over 50 initial conditions and in the case of $L R_{A N N} 25$ ANNs. The shaded region depicts the standard deviation of the RMSE.

Next we examine the effect of the ANN on a 48-hour forecast. Here we compare a LR simulation with $\left(L R_{A N N}\right)$ and without $(L R)$ the use of the ANN. Both simulations start from the same initial conditions as the model truth. This is repeated for 50 initial conditions, each 2 hours apart. The results in terms of RMSE with respect to the model truth are presented in Figure 

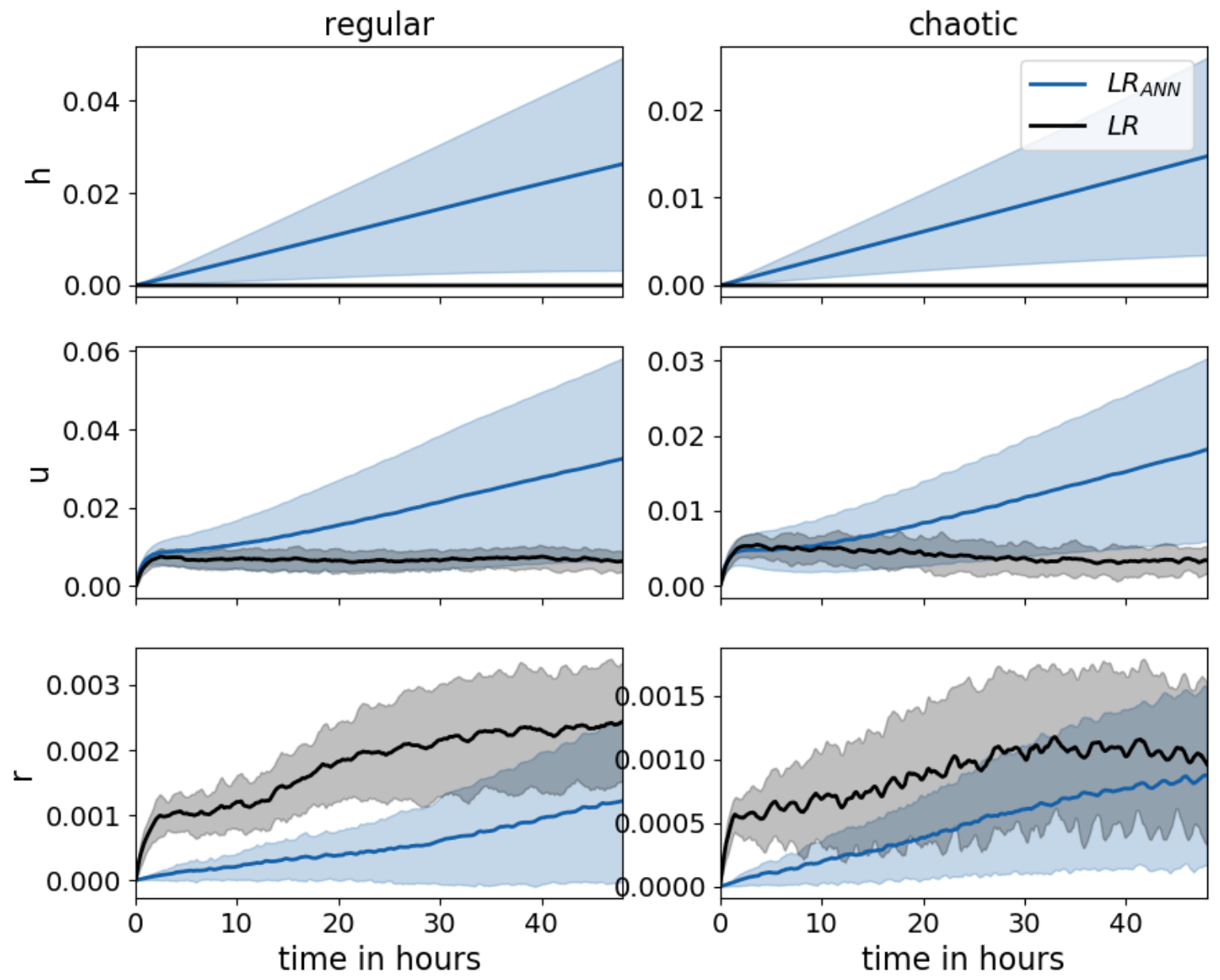

Figure 5. Same as Figure 4, but for the absolute error of the spatial mean of the variables.

4. Note that the shaded region for $L R_{A N N}$ includes the variability due to the ANN and the initial conditions. The RMSEs corresponding to the regular case are higher than for the chaotic case. This is because the regular case exhibits a repeating pattern of long-lived, high amplitude convective events (not shown). In comparison, the chaotic case produces short-lived perturbations with very small amplitude (not shown), leading to smaller climatological variability.

For both orographies the ANN has a clear positive effect on the forecast until the error of $L R$ saturates, after which the error of $L R_{A N N}$ continues to grow. For the chaotic case this leads to a detrimental impact of the ANN after about 30 hours. It is not surprising that $L R_{A N N}$ deteriorates as the forecast lead time increases, since the ANNs are not perfect (as opposed to the data they were trained on) and the resulting errors accumulate over time, leading to biases. This is clearly visible in Figure 5, where 
it is seen that the domain mean error of $h$ and $r$ diverges, in contrast to $L R$. It is worth noting that the domain mean error of

$r$ for $L R$ is the result of a negative bias in the amount of rain produced, caused by the coarse graining of the orography (not shown). This bias is partially (sometimes over) corrected by the ANNs. The divergence of the domain mean error of $h$ is the result of applying ANNs that, in contrast to the model, do not conserve mass. This leads to accumulated mass errors, causing biases in the wind field due to momentum conservation and a change in probability for the fluid to rise above $H_{c}$ and $H_{r}$. We therefore investigate if reducing the mass error, by adding a penalty term to the loss function of the ANN, can increase the forecast skill further.

\subsection{ANN with mass conservation in a weak sense}

We have trained ANNs with mass conservation weightings $w_{\text {mass }}=1,10,100,1000$. These weightings result in a contribution to the loss function of roughly $0.2 \%, 0.7 \%, 2 \%$ and $5 \%$ throughout the training process respectively (not shown). Note that the ANNs presented in the previous section correspond to $w_{\text {mass }}=0$.

Figures 6 and 7 show the single time step predictions improvements for both orographies. Clearly, the mass conservation penalty term in the loss function has the desired effect of reducing the mass error for both orographies, though for the regular case a weighting of $w_{\text {mass }}>1$ is necessary. As hypothesized, reducing the mass error also has a positive effect on the domain mean error of the wind $u$. The standard deviation of the bias is much larger than the mean bias for all variables and both orographies. We believe that the mean bias would go to zero as the sample size (which is now only 25) increases. This indicates that single ANNs do not have a preference for either a positive or negative bias. The decrease of standard deviation of the bias as $w_{\text {mass }}$ increases confirms the correlation between the mass error in $h$ and the wind bias. Contrary to what we expected, the RMSE does not generally go up when $w_{\text {mass }}$ is increased. It even seems to go down for all variables. We do see the RMSE for $w_{\text {mass }}=1000$ going up for the regular case, which indicates that there is threshold after which the penalty terms becomes detrimental to the RMSE. For the regular case this threshold lies between $100<W_{\text {mass }}<1000$ and for the chaotic case it lies above $w_{\text {mass }}>1000$, assuming it exists at all.

Figure 8 presents the mean RMSE of the 48-hour forecasts for all weightings. The weak mass conservation constraint has the desired effect on the forecast skill. For the chaotic case, about 10 hours in forecast quality is gained. For the regular case the exact number is unclear since the RMSE is still lower than $L R$ and has not yet saturated after 48 hours. However we can say that it is at least 30 hours. As hypothesized, Figure 9 indicates that the divergence of the domain mean error of the wind $u$ is delayed as the weighting $w_{\text {mass }}$ is increased. This in turn positively affects the domain mean of the rain $r$. To support these claims we look at Figure 10, which shows the correlation between the bias in $h$ and the bias in wind $u$ and rain $r$ respectively. The wind bias becomes almost completely anticorrelated to the mass bias as the forecast lead time increases. In the single step predictions we did not detect a correlation between the mass bias and the rain bias. Figure 10 however demonstrates that this correlation is established after a few time steps, likely when the change in probability of crossing the rain threshold resulting from the mass bias has taken effect. We also note that the smaller $w_{\text {mass }}$, the stronger the correlation. We hypothesize that as the mass bias weakens, other causes for introducing domain mean biases in the wind and rain field become more significant. Such other causes may for example depend on the orography, or the state of $u$ and $r$. 

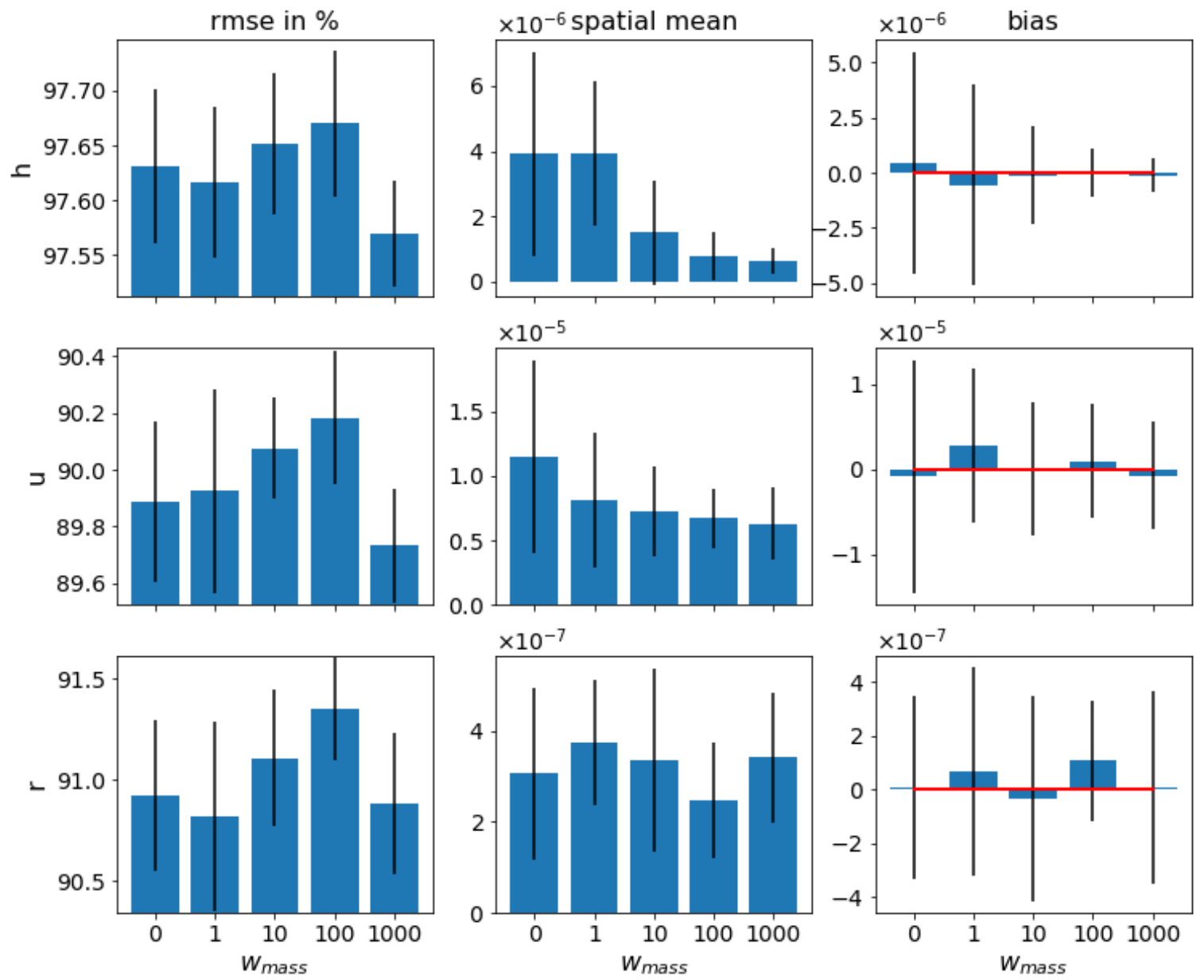

Figure 6. Mean RMSE expressed in improvement as in Figure 3 (left), absolute error of the spatial mean (middle), and bias (right) of the validation data for the different weightings (x-axis) and the respective model variables (rows) for the regular case. Error bars indicate the standard deviation and the red lines in the right panel indicate the zero line.

Next we look at the variability of the forecast errors in Figure 11. Here we look at the variability due to the initial conditions, the ANN, and the combination of both. For small weightings the variability due to the ANN seems to dominate the total variability. However, as the weighting increases, the variability due to the initial conditions takes over. This again confirms the benefits of adding the mass penalty term to the loss function, as it demonstrates decrease in sensitivity of the forecast to the training process of the ANN. 

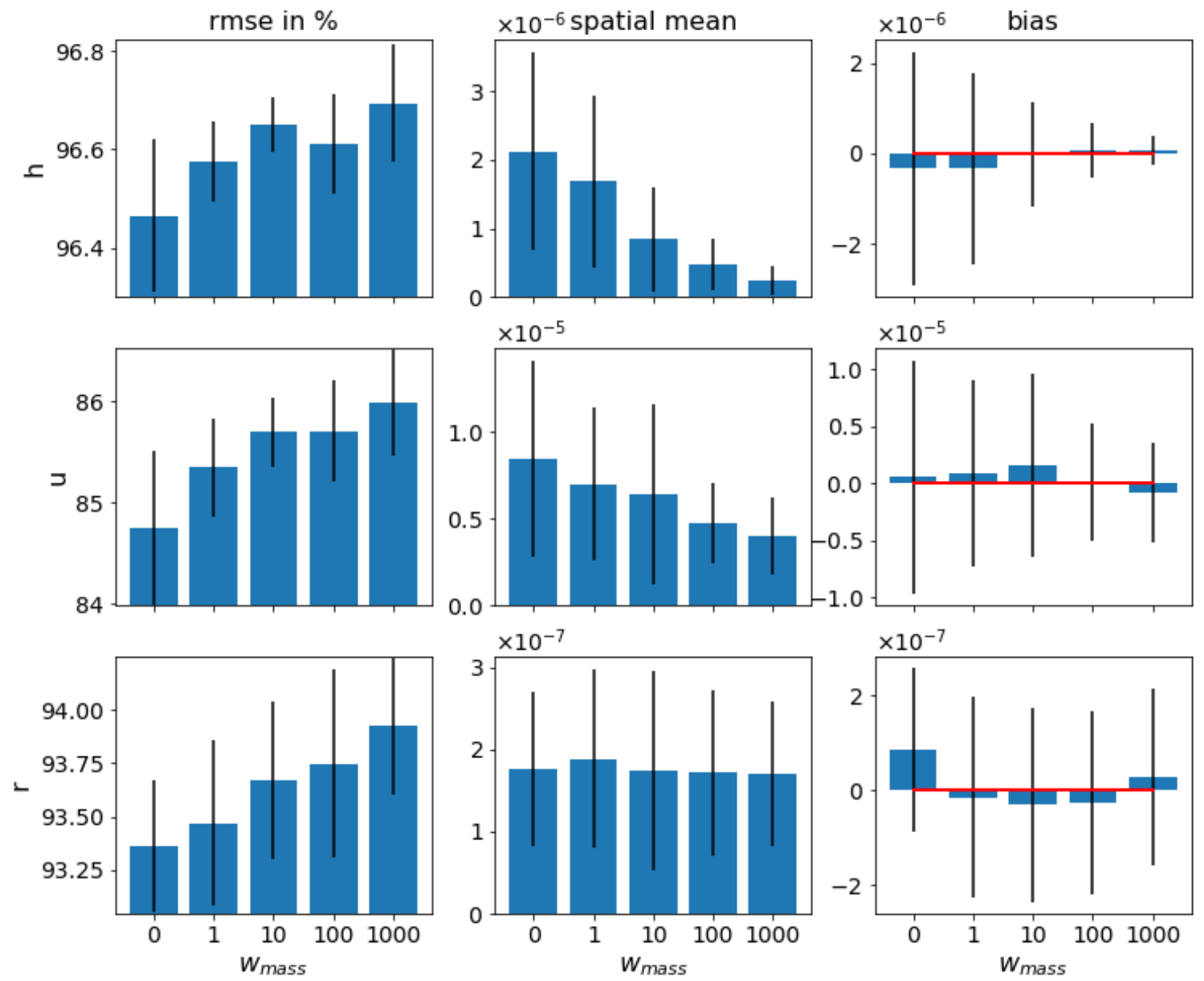

Figure 7. Same as Figure 6, but for the chaotic case.

Based on the subjective interpretation of the human brain of a hand full of animations of the forecast evolution, it appears that convective events produced in the LR run are wider and shallower than in the coarse grained HR run, likely due to the smoothening of the orography. As discussed before, this results in a lack of rain mass, but also, via conservation of momentum, a drift in the wind field. The convective events in the LR simulations are therefore also increasingly misplaced as the forecast lead time increases. The ANNs are capable of sharpening the gradients of the convective events, leading to highly accurate forecasts of convective events up to 5-10 hours. After this, spurious, missed and misplaced events start to occur, although the forecast skill remains significant up to at least 24 hours, in contrast to the LR simulations, where the forecast skill dissolves 

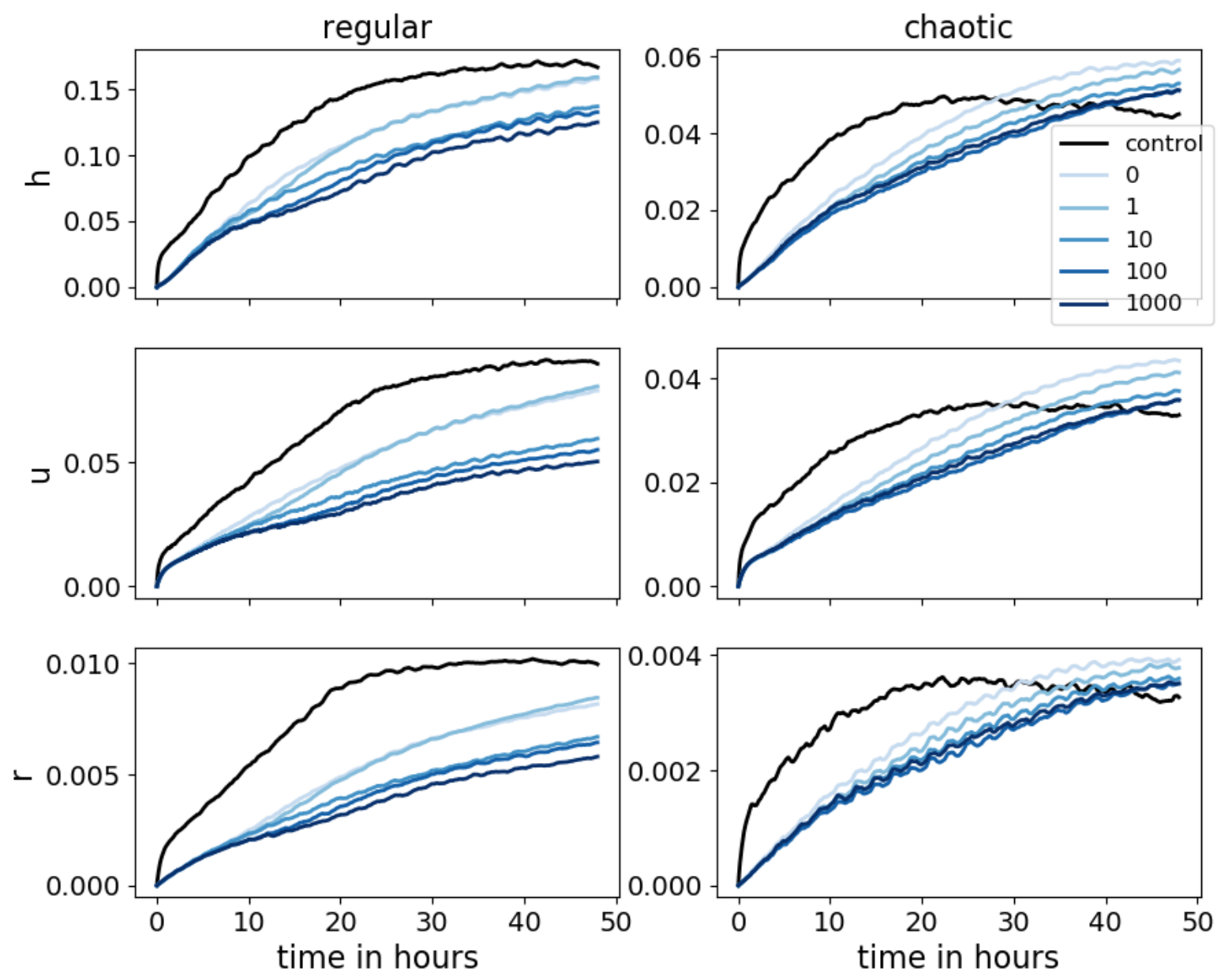

Figure 8. RMSE evolution of 48-hour forecasts for the respective model variables of $L R$ (black) and $L R_{A N N}$ for the different weightings (blues), averaged over 50 initial conditions and in the case of $L R_{A N N} 25$ ANNs.

after just a few hours. A snapshot of the state for the chaotic case is presented in Figure 12. Here, the correlations between the 3 variables is nicely visible. Between grid points 100 and 125, the fluid height $h$ of $L R$ is exceeding both the convective initiation and rain threshold, in contrast to the other simulations. As a result, $L R$ has produced spurious rain mass at this location and the wind is underestimated. Directly right of this spurious convective event in $L R$, the roles are exchanged: The fluid height of $L R$ does not reach the thresholds, and therefore lacks rain and overestimates the wind speed. The same correlations are discernible between grid points 50 and 75 , where the ANN with $w_{\text {mass }}=0$ slightly overestimates the fluid height. 

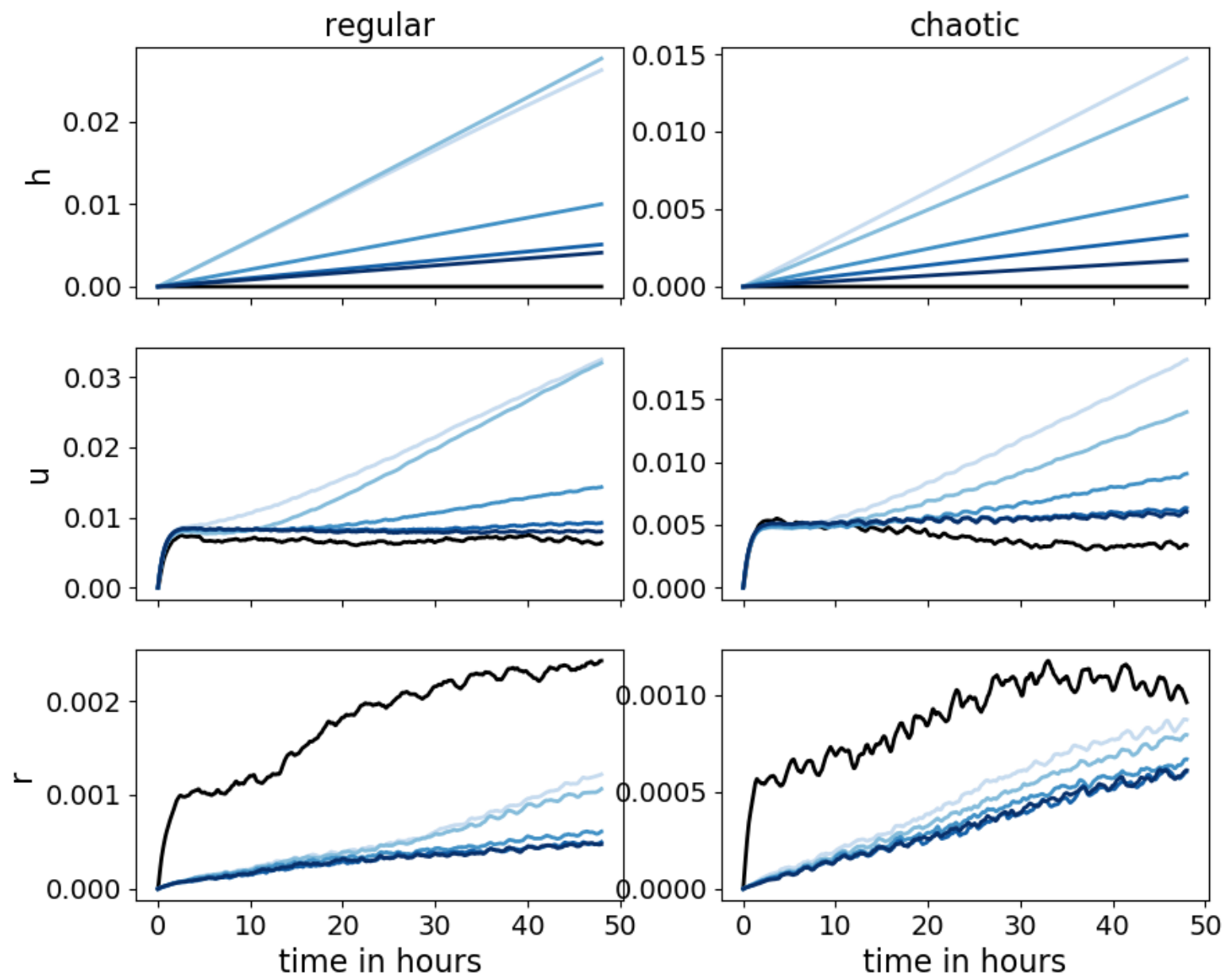

Figure 9. As Figure 8, but for the absolute error of the spatial mean.

\section{Conclusions}

In this paper we evaluated the feasibility of using an ANN to correct for model error in the gray zone, where important features occur on scales comparable to the model resolution. The model that was used in our idealized setup mimics key aspects of convection such as conditional instability triggered by orography and resulting convective events including rain. As such, this model is representative for fully complex convective scale numerical weather prediction models and in particular the corresponding errors due to unresolved scales in the gray zone. We considered two cases, each with a different realization of the orography, leading to two fairly different regimes. One where the convective events are large and long-lived, and one where the 

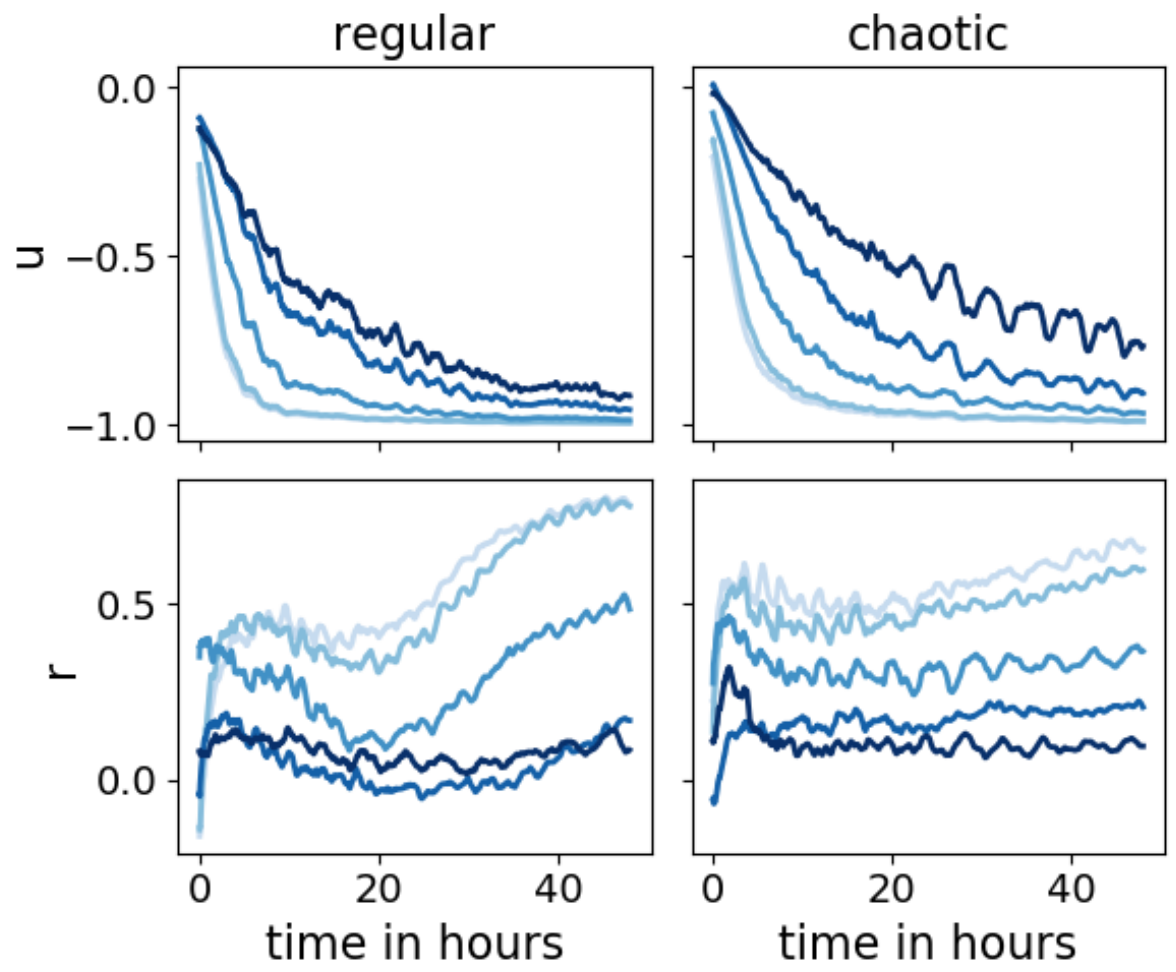

Figure 10. Correlation of the different weighting (blues) between the bias of $h$ and the bias of $u$ (top row) and $r$ (bottom row) for the regular (left panel) and the chaotic (right panel) case. See Figure 8 for the legend.

convective events are small and short-lived. We refer to the former case as regular and the latter case as chaotic. We showed that the ANNs are capable of accurately sharpening gradients where necessary in both cases to prevent the flattening of convective events that is caused by the low resolution model's inability to resolve fine scales. For the regular case, the RMSE is still significantly lower than the low resolution simulation $(L R)$ after 48 hours. For the chaotic case, the RMSE surpasses $L R$ after about 30 hours. Since the ANNs are not perfect, their errors accumulate over time, deteriorating the forecast skill. In particular, the accumulated mass error causes biases which are not present in $L R$, because the model conserves mass exactly. We therefore investigated the effects of adding a term to the loss function of the ANN's training process to penalize mass conservation violation. We found that reducing the mass error, reduces the biases in the wind and rain field, yielding better forecasts up to 10 hours for the chaotic and 30 hours for regular case in terms of RMSE. Such positive effect of mass conservation was also found in for example Zeng et al. (2017); Ruckstuhl and Janjić (2018); Ruckstuhl et al. (2021). Furthermore, we showed that including the penalty term in the loss function reduces the sensitivity of the model forecasts to the training process of the ANN.

While these results are encouraging, there are some issues to consider when applying this method to operational configurations. On a technical level, the generation of the training data and the training of the ANN can be costly and time consuming 

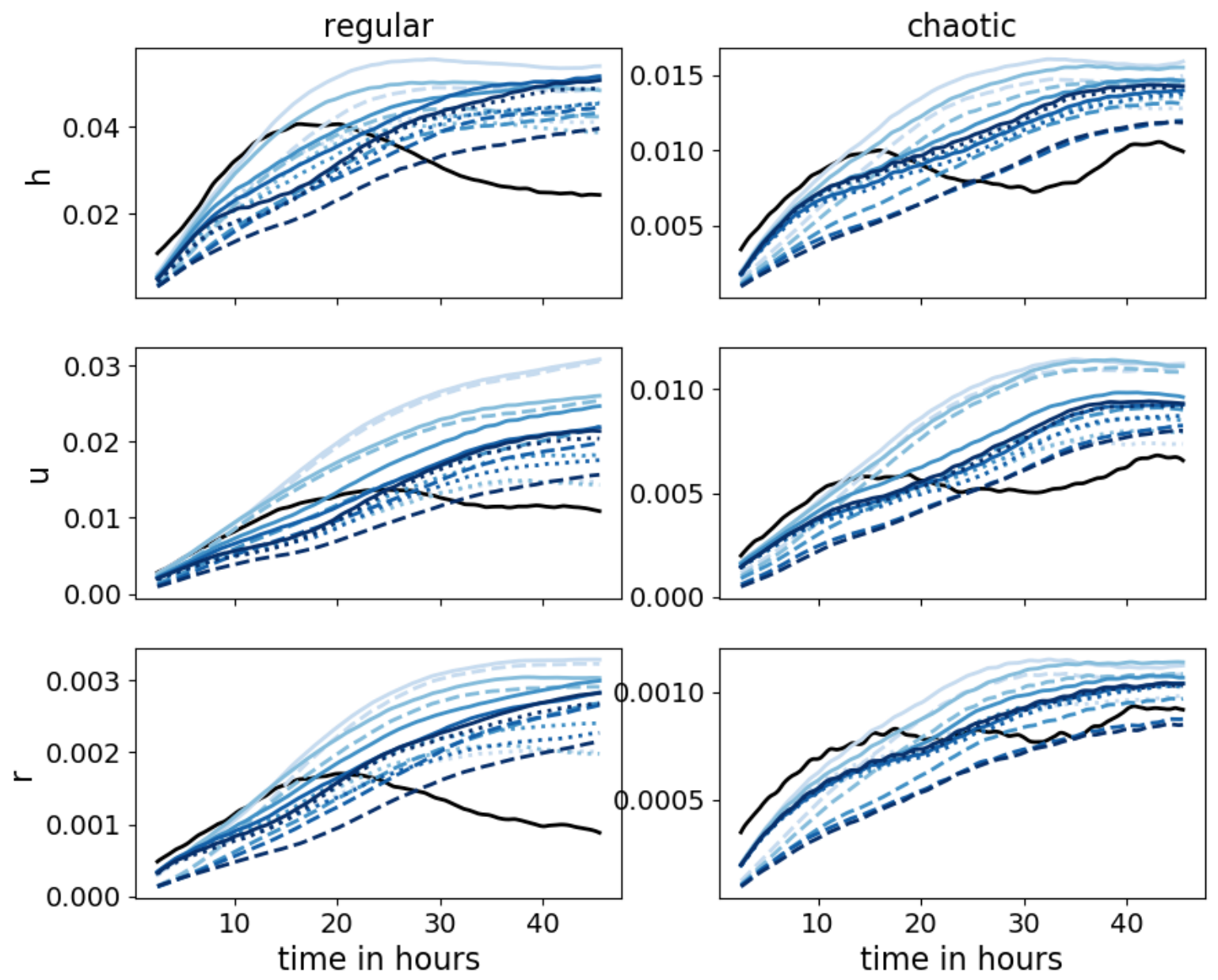

Figure 11. Evolution of the standard deviation for the different weightings (blues) of the RMSE over both ANNs and initial conditions (solid), over ANNs only and averaged over initial conditions (dashed), and over initial conditions only and averaged over ANNs (dotted), for the regular (left panel) and the chaotic (right panel) case. See Figure 8 for the legend.

due to the requirement of sufficient HR data and the cumbersome exercise of tuning the ANN. The latter is a known problem that can be minimized through clever iteration of tested ANN settings, but cannot be fully avoided. Depending on the costs of generating HR data, it could be considered to use observations instead, as done by Brajard et al. (2021). They use data assimilation to generate HR data from available sparse and noisy observations. Aside from saving computational costs by replacing HR simulations with data assimilation, it might offer an advantage on a different issue as well: the effect of other model error. In contrast to what was assumed in this paper, in reality not all model error stems from unresolved scales. By using observations 

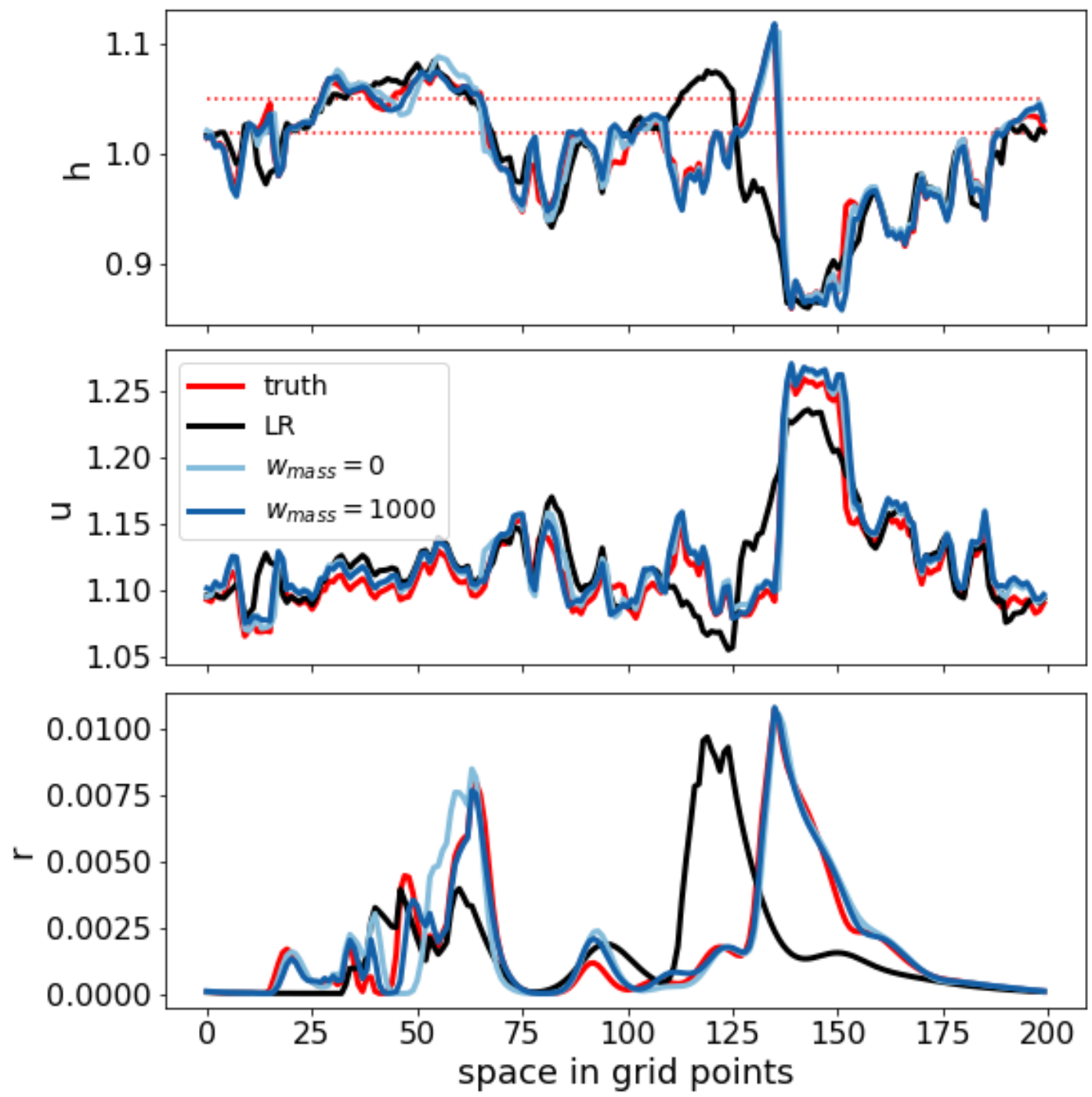

Figure 12. Snapshot of the state variables for the chaotic case of a 5-hour forecast for the truth (red), $L R$ (black), and $L R_{A N N}$ corresponding to weightings $w_{\text {mass }}=0$ (light blue) and $w_{\text {mass }}=1000$ (dark blue).

of the true state of the atmosphere, all model error is accounted for by the trained ANN. On the other hand, the training data contains the errors inherited from data assimilation. It is not clear which error source is more important and therefore both approaches are worthwhile investigating. Not only to improve model forecasts, but also to gain more insight in the model error itself and its comparison to errors stemming from data assimilation. 
https://doi.org/10.5194/npg-2021-20

Preprint. Discussion started: 17 May 2021

(c) Author(s) 2021. CC BY 4.0 License.

(c) (i)

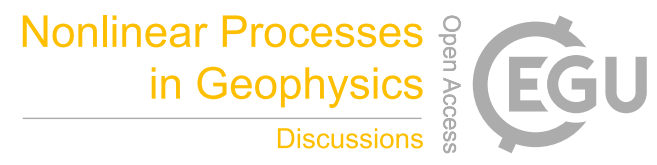

Code availability. The provided source code (https://doi.org/10.5281/zenodo.4740252, Kriegmair et al., 2020) includes the necessary scripts to produce the data.

Author contributions. RK produced the source code. RK and YR ran experiments and visualized results. SR provided expertise on neural networks. GC provided expertise on convective scale dynamics. All authors contributed to the scientific design of the study, the analysis of the numerical results and the writing of the manuscript.

Competing interests. The authors declare that they have no conflict of interest.

Acknowledgements. The research leading to these results has been done within the Transregional Collaborative Research Center SFB/TRR 165 "Waves to Weather" (www.wavestoweather.de) funded by the German Research Foundation (DFG). 
https://doi.org/10.5194/npg-2021-20

Preprint. Discussion started: 17 May 2021

(c) Author(s) 2021. CC BY 4.0 License.

(c) (i)

\section{References}

Bocquet, M., Brajard, J., Carrassi, A., and Bertino, L.: Bayesian inference of chaotic dynamics by merging data assimilation, machine learning and expectation-maximization, Foundations of Data Science, 2, 55-80, https://doi.org/10.3934/fods.2020004, 2020.

Bolton, T. and Zanna, L.: Applications of Deep Learning to Ocean Data Inference and Subgrid Parameterization, Journal of Advances in Modeling Earth Systems, 11, 376-399, https://doi.org/10.1029/2018MS001472, 2019.

Brajard, J., Carrassi, A., Bocquet, M., and Bertino, L.: Combining data assimilation and machine learning to emulate a dynamical model from sparse and noisy observations: A case study with the Lorenz 96 model, Journal of Computational Science, 44, 101 171, https://doi.org/https://doi.org/10.1016/j.jocs.2020.101171, 2020a.

Brajard, J., Carrassi, A., Bocquet, M., and Bertino, L.: Combining data assimilation and machine learning to infer unresolved scale parametrization, Philosophical Transactions of the Royal Society A: Mathematical, Physical and Engineering Sciences, 379, 20200 086, https://doi.org/10.1098/rsta.2020.0086, 2021.

Brenowitz, N. D. and Bretherton, C. S.: Spatially Extended Tests of a Neural Network Parametrization Trained by Coarse-Graining, Journal of Advances in Modeling Earth Systems, 11, 2728-2744, https://doi.org/10.1029/2019MS001711, 2019.

Brunton, S. L., Proctor, J. L., and Kutz, J. N.: Discovering governing equations from data by sparse identification of nonlinear dynamical systems, Proceedings of the National Academy of Sciences, 113, 3932-3937, https://doi.org/10.1073/pnas.1517384113, 2016.

Bryan, G. H., Wyngaard, J. C., and Fritsch, J. M.: Resolution Requirements for the Simulation of Deep Moist Convection, Monthly Weather Review, 131, 2394 - 2416, https://doi.org/10.1175/1520-0493(2003)131<2394:RRFTSO>2.0.CO;2, 2003.

Chow, F. K., Schär, C., Ban, N., Lundquist, K. A., Schlemmer, L., and Shi, X.: Crossing Multiple Gray Zones in the Transition from Mesoscale to Microscale Simulation over Complex Terrain, Atmosphere, 10, https://doi.org/10.3390/atmos10050274, 2019.

Dueben, P. D. and Bauer, P.: Challenges and design choices for global weather and climate models based on machine learning, Geoscientific Model Development, 11, 3999-4009, https://doi.org/10.5194/gmd-11-3999-2018, 2018.

Fablet, R., Ouala, S., and Herzet, C.: Bilinear Residual Neural Network for the Identification and Forecasting of Geophysical Dynamics, in: 2018 26th European Signal Processing Conference (EUSIPCO), pp. 1477-1481, https://doi.org/10.23919/EUSIPCO.2018.8553492, 2018.

Faranda, D., Vrac, M., Yiou, P., Pons, F. M. E., Hamid, A., Carella, G., Ngoungue Langue, C., Thao, S., and Gautard, V.: Boosting performance in machine learning of geophysical flows via scale separation, Nonlinear Processes in Geophysics Discussions, 2020, 1-30, https://doi.org/10.5194/npg-2020-39, 2020.

Honnert, R., Efstathiou, G. A., Beare, R. J., Ito, J., Lock, A., Neggers, R., Plant, R. S., Shin, H. H., Tomassini, L., and Zhou, B.: The Atmospheric Boundary Layer and the "Gray Zone" of Turbulence: A Critical Review, Journal of Geophysical Research: Atmospheres, 125, e2019JD030 317, https://doi.org/https://doi.org/10.1029/2019JD030317, e2019JD030317 10.1029/2019JD030317, 2020.

Jeworrek, J., West, G., and Stull, R.: Evaluation of Cumulus and Microphysics Parameterizations in WRF across the Convective Gray Zone, Weather and Forecasting, 34, 1097 - 1115, https://doi.org/10.1175/WAF-D-18-0178.1, 2019.

Jones, T. R. and Randall, D. A.: Quantifying the limits of convective parameterizations, Journal of Geophysical Research: Atmospheres, 116, https://doi.org/10.1029/2010JD014913, 2011.

Kent, T., Bokhove, O., and Tobias, S.: Dynamics of an idealized fluid model for investigating convective-scale data assimilation, Tellus A: Dynamic Meteorology and Oceanography, 69, 1369332, https://doi.org/10.1080/16000870.2017.1369332, 2017. 
https://doi.org/10.5194/npg-2021-20

Preprint. Discussion started: 17 May 2021

(c) Author(s) 2021. CC BY 4.0 License.

\section{(c) (i)}

Krasnopolsky, V. M., Fox-Rabinovitz, M. S., and Belochitski, A. A.: Using ensemble of neural networks to learn stochastic convection parameterizations for climate and numerical weather prediction models from data simulated by a cloud resolving model, Adv Artif Neural Syst, 2013, http://dx.doi.org/10.1155/2013/485913, 2013.

O’Gorman, P. A. and Dwyer, J. G.: Using Machine Learning to Parameterize Moist Convection: Potential for Modeling of Climate, Climate Change, and Extreme Events, Journal of Advances in Modeling Earth Systems, 10, 2548-2563, https://doi.org/https://doi.org/10.1029/2018MS001351, 2018.

Pathak, J., Hunt, B., Girvan, M., Lu, Z., and Ott, E.: Model-Free Prediction of Large Spatiotemporally Chaotic Systems from Data: A Reservoir Computing Approach, Phys. Rev. Lett., 120, 024 102, https://doi.org/10.1103/PhysRevLett.120.024102, 2018.

Randall, D., Khairoutdinov, M., Arakawa, A., and Grabowski, W.: Breaking the Cloud Parameterization Deadlock, Bulletin of the American Meteorological Society, 84, 1547-1564, https://doi.org/10.1175/BAMS-84-11-1547, 2003.

Randall, D. A.: Beyond deadlock, Geophysical Research Letters, 40, 5970-5976, https://doi.org/10.1002/2013GL057998, 2013.

Rasp, S.: Coupled online learning as a way to tackle instabilities and biases in neural network parameterizations: general algorithms and Lorenz 96 case study (v1.0), Geoscientific Model Development, 13, 2185-2196, https://doi.org/10.5194/gmd-13-2185-2020, 2020.

Rasp, S., Pritchard, M. S., and Gentine, P.: Deep learning to represent subgrid processes in climate models, Proceedings of the National Academy of Sciences, 115, 9684-9689, https://doi.org/10.1073/pnas.1810286115, 2018.

Ruckstuhl, Y. and Janjić, T.: Parameter and state estimation with ensemble Kalman filter based algorithms for convective-scale applications, Quart .J. Roy. Meteorol. Soc, 144, 826-841, https://doi.org/10.1002/qj.3257, 2018.

Ruckstuhl, Y., Janjić, T., and Rasp, S.: Training a convolutional neural network to conserve mass in data assimilation, Nonlinear Processes in Geophysics, 28, 111-119, https://doi.org/10.5194/npg-28-111-2021, 2021.

Scher, S.: Toward Data-Driven Weather and Climate Forecasting: Approximating a Simple General Circulation Model With Deep Learning, Geophysical Research Letters, 45, 12,616-12,622, https://doi.org/https://doi.org/10.1029/2018GL080704, 2018.

Stensrud, D.: Parameterization Schemes: Keys to Understanding Numerical Weather Prediction Models, Cambridge University Press, https: //books.google.de/books?id=kkZ0AgAAQBAJ, 2009.

Wagner, A., Heinzeller, D., Wagner, S., Rummler, T., and Kunstmann, H.: Explicit Convection and Scale-Aware Cumulus Parameterizations: High-Resolution Simulations over Areas of Different Topography in Germany, Monthly Weather Review, 146, 1925 - 1944, https://doi.org/10.1175/MWR-D-17-0238.1, 2018.

Würsch, M. and Craig, G. C.: A simple dynamical model of cumulus convection for data assimilation research, Meteorologische Zeitschrift, 23, 483-490, https://doi.org/10.1127/0941-2948/2014/0492, 2014.

Yuval, J. and O'Gorman, P. A.: Stable machine-learning parameterization of subgrid processes for climate modeling at a range of resolutions, Nature communications, 11, 1-10, 2020.

295 Zeng, Y., Janjić, T., Ruckstuhl, Y., and Verlaan, M.: Ensemble-type Kalman filter algorithm conserving mass, total energy and enstrophy, Quarterly Journal of the Royal Meteorological Society, 143, 2902-2914, https://doi.org/10.1002/qj.3142, 2017. 